\title{
CROWN-LEVEL TREE SPECIES CLASSIFICATION USING INTEGRATED AIRBORNE HYPERSPECTRAL AND LIDAR REMOTE SENSING DATA
}

\author{
Zhihui Wang ${ }^{1,2}$, Jie Wu ${ }^{3, *}$, Yuanjian Wang ${ }^{1,2}$, Xiangbing Kong ${ }^{1,2}$, Hongzhe Bao ${ }^{1,2}$, Yongxin $\mathrm{Ni}^{1,2}$, Li Ma ${ }^{1,2}$, Chengran Jin ${ }^{1,2}$
}

\author{
${ }^{1}$ Yellow River Institute of Hydraulic Research, Yellow River Conservancy Commission, Zhengzhou, China - wzh8588@aliyun.com \\ ${ }^{2}$ Key Laboratory of the Loess Plateau Soil Erosion and Water Process and Control, Ministry of Water Resources, Zhengzhou, China, \\ ${ }^{3}$ Hydrology and Water Resources Institute, Hohai University, Nanjing, China - 936992107@qq.com
}

\author{
Commission III, WG III/1-Poster
}

KEY WORDS: Tree species classification, Crown-scale spectral, Crown-scale structure, LiDAR, Hyperspectral

\begin{abstract}
:
Mapping tree species is essential for sustainable planning as well as to improve our understanding of the role of different trees as different ecological service. However, crown-level tree species automatic classification is a challenging task due to the spectral similarity among diversified tree species, fine-scale spatial variation, shadow, and underlying objects within a crown. Advanced remote sensing data such as airborne Light Detection and Ranging (LiDAR) and hyperspectral imagery offer a great potential opportunity to derive crown spectral, structure and canopy physiological information at the individual crown scale, which can be useful for mapping tree species. In this paper, an innovative approach was developed for tree species classification at the crown level. The method utilized LiDAR data for individual tree crown delineation and morphological structure extraction, and Compact Airborne Spectrographic Imager (CASI) hyperspectral imagery for pure crown-scale spectral extraction. Specifically, four steps were include: 1) A weighted mean filtering method was developed to improve the accuracy of the smoothed Canopy Height Model (CHM) derived from LiDAR data; 2) The marker-controlled watershed segmentation algorithm was, therefore, also employed to delineate the tree-level canopy from the CHM image in this study, and then individual tree height and tree crown were calculated according to the delineated crown; 3) Spectral features within $3 \times 3$ neighborhood regions centered on the treetops detected by the treetop detection algorithm were derived from the spectrally normalized CASI imagery; 4) The shape characteristics related to their crown diameters and heights were established, and different crown-level tree species were classified using the combination of spectral and shape characteristics. Analysis of results suggests that the developed classification strategy in this paper $(\mathrm{OA}=85.12 \%, \mathrm{Kc}=0.90)$ performed better than LiDAR-metrics method $(\mathrm{OA}=79.86 \%, \mathrm{Kc}=0.81)$ and spectral-metircs method $(\mathrm{OA}=71.26, \mathrm{Kc}=0.69)$ in terms of classification accuracy, which indicated that the advanced method of data processing and sensitive feature selection are critical for improving the accuracy of crown-level tree species classification.
\end{abstract}

\section{INTRODUCTION}

Globally, over 10 million $\mathrm{km}^{2}$ of agricultural land has a tree cover greater than $10 \%$ (Li et al., 2003). Agroforestry has been much advocated and practiced in China and around the world in the past five decades because agroforestry combining agricultural and forestry techniques is able to create more diverse, productive, profitable, healthy and sustainable land use systems (Zomer et al., 2009). The forests that grow in these areas have significantly positive effects on the fertility of the soil and the productivity of the agricultural land, mitigating the impacts of climate variability and change, conserving biodiversity and improving air and water quality (Hernandez et al., 2012). In addition, woody trees on agricultural land has the potential for carbon (C) sequestration while providing many economic, social, and ecological benefits. Therefore, mapping tree species is essential for sustainable planning as well as to improve our understanding of the role of different trees in the agroforestry ecosystem (George et al., 2014).
Hyperspectral data is considered effective for mapping tree species as it can measure subtle variability in spectral reflectance from leaf to crown scales, largely due to their very high spectral resolution and wide range of electromagnetic spectrum (George et al., 2014). As a result, hyperspectral data have been widely applied in mapping tree species in different kinds of forest ecosystems. Species classification has been usually carried out at pixel and crown levels. Pixel-level classifications have also been employed for tree species classification due to its easiness for implementation and interpretation (Clark and Roberts, 2012). However, pixel-level classification ignores the negative impact of the mixed pixel problem (Lu and Weng, 2004), which may lead to the "salt and pepper" effect in the final classification result (Yu et al., 2006). Crown-level classification is increasingly demanded in comparison with pixel level, and sub-pixel level classifications. Species at the individual tree level is often considered as the management unit in practical forest application (Dalponte et al., 2012), and the object-oriented method can overcome the limitations of pixel-level classification, such as spatial

\footnotetext{
* Corresponding author: Jie Wu - 936992107@qq.com
} 
heterogeneity and mixed-pixel problem (Ke et al., 2014). Several methods have been developed for crown-level species classification. (Clark et al., 2005) linearly averaged the pixel spectra within a manually-delineated crown area as the crownscale spectra for tropical rain forest species classification, and the highest crown-scale classification accuracy of seven species reached $92 \%$ with the linear discriminant analysis (LDA) and 30 optimal bands. (Alonzo et al.,2014) integrated Hyperspectral imagery with LiDAR data for mapping 29 tree species in Santa Barbara, California, USA, and an overall accuracy of $83.4 \%$ was reported.

However, crown-level tree species automatic classification is a challenging task due to crown-scale spectra have lower purity due to the interference of mixed pixel problem and double-side illumination problem (Shang and Chisholm, 2014; Zhang and Qiu, 2012). This lower purity of crown spectra, therefore, may contribute to the lower classification of tree species at the crown level. In order to address this issue, we developed an innovative method using the combination of spectral and shape characteristics of different tree species, which may potentially improve tree species classification accuracy. Specific aims of this paper are: 1) to segment individual crowns using LiDAR derived canopy height model, 2) to calculate the crown-scale spectra from CASI hyperspectral imagery using spectral normalization method, 3) to classify tree species by applying decision tree classifier based on spectral and shape characteristics. In order to assess the improvement of this method in classifying tree species, the LiDAR-metrics and spectral-metrics approaches will be carried out for a comparative analysis.

\section{MATERIALS}

\subsection{Study area}

The study was carried out in an irrigated oasis district in the middle reaches of the Heihe River Basin, China. The Heihe River originates in the Qilian Mountains and flows through Qinghai, Gansu and Inner Mongolia; it is the second longest inland river (it eventually flows into the desert) in China. The study area encompassed the area between longitudes $97^{\circ} 24^{\prime}$ and $102^{\circ} 10^{\prime} \mathrm{E}$ and latitudes $37^{\circ} 41^{\prime}$ and $42^{\circ} 42^{\prime} \mathrm{N}$ (Figure 1). The middle reaches of the Heihe River are dominated by a temperate arid climate with an annual precipitation of $113.8 \mathrm{~mm}-183.5 \mathrm{~mm}$, an annual potential evapotranspiration of $1400-2800 \mathrm{~mm}$, an annual sunshine amount of 3000-4000 hours and an average annual temperature of $6^{\circ} \mathrm{C}-8^{\circ} \mathrm{C}$. The oasis supports irrigated agriculture dominated by a variety of planted crops and agricultural protection forests that have a large internal spatial heterogeneity.

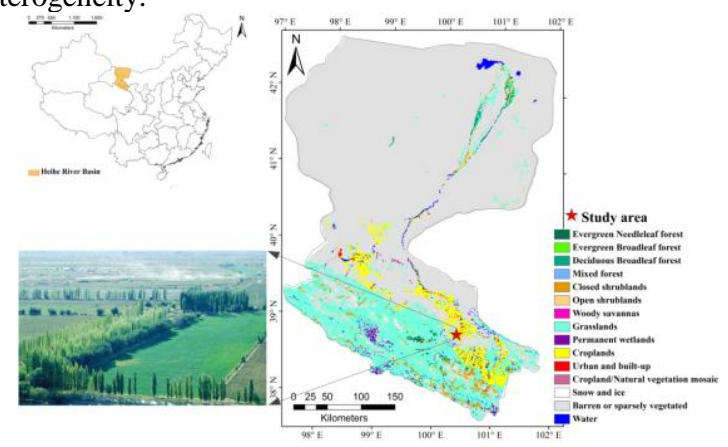

Figure 1. The location of the study area in the middle reaches of the Heihe River Basin.

\subsection{Airborne CASI and LiDAR data acquisition}

CASI-1500 was employed to record hyperspectral imagery on 29 June 2012. The hyperspectral images had 48 spectral bands centered between $382.5 \mathrm{~nm}$ and $1055.5 \mathrm{~nm}$, a spatial resolution of $1.0 \mathrm{~m}$, a spectral resolution of $7.2 \mathrm{~nm}$ ( $\mathrm{Li}$ et al., 2003). The small-footprint LiDAR point cloud data used in this study were provided by the HiWATER program (Li et al., 2003).

\subsection{Ground survey}

A ground survey was carried out during July 2012 by the HiWATER program (Li et al., 2003; Cheng et al, 2014). First, the distribution of woody trees and tree species were recorded. According to the field survey, poplar (Populus spp.), willow (Salix babylonica L.) and Chinese scholar tree (Sophora japonica Linn.) were the three dominant species in the study area, with poplar being the most dominant. 867 trees in 59 field sites were in-situ surveyed, including 556 poplar trees, 165 willow tree and 146 scholar trees, as illustrated in the Figure 2. Within each plot, the vegetation structure parameters of tree height and crown diameter were recorded.

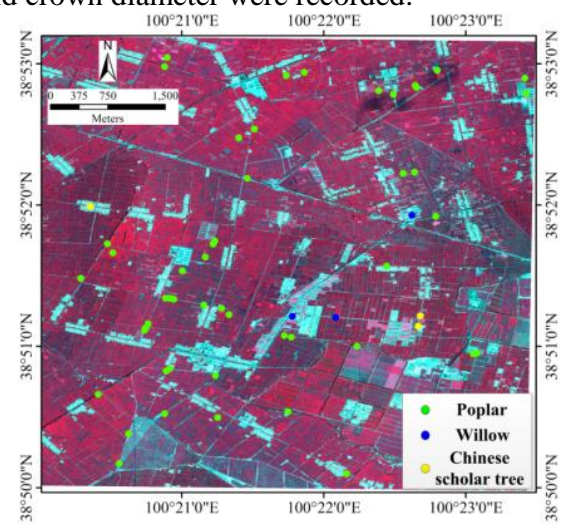

Figure 2. Spatial distribution of the measured trees for three tree species: poplar, willow and Chinese scholar.

\section{METHODOLOGY}

\subsection{Extraction of crown-level structural parameters}

\subsubsection{Generation of woody canopy height model:}

In the case of this area of agroforestry, the land cover types include tree, cropland, buildings, roads and bare land, and the heights of a large number of buildings are similar to those of the trees. Therefore, it is impossible accurately to distinguish between the canopy and non-canopy pixels using only a height threshold derived from the NDSM. In this study, the spectral characteristics of trees derived from CASI data were also employed to make a further classification of pixels into (a) tree canopy pixels and (b) non-canopy pixels that had a similar height as those containing trees.

In this study, the CHM was developed by integrating the morphological crown control (MCC) method(Zhao et al., 2013) and the spectral index threshold. The rules used to produce the CHM for the woody trees in agroforestry are described by Eq. (1).

$$
C_{\text {tree }}(i, j)=\left\{\begin{array}{l}
\operatorname{Mean}\left(R V I_{3 \times 3}\right)>T_{R V I} \\
\operatorname{NDSM}_{\text {close }}(i, j)>T_{\text {close }}
\end{array}\right.
$$

Where $\mathrm{C}_{\text {tree }}$ is the CHM of the trees. Mean $\left(\mathrm{RVI}_{3 \times 3}\right)$ is the mean value of the simple ratio vegetation index (RVI) calculated using the red and near-infrared bands of the CASI image in the $3 \times 3$-pixel neighborhood region of the pixel $(i, j)$. A $3 \times 3$ neighborhood was used to overcome the positional bias between the NDSM and the CASI hyperspectral image. $\mathrm{T}_{\mathrm{RVI}}$ is 
the RVI threshold, which was set as 2.0 in this study based on the results of repeated trials. NDSMclose is the NDSM smoothed by the morphological closing operator-according to earlier research(Zhao et al., 2013). $\mathrm{T}_{\text {close }}$ is the threshold used to determine tree crown pixels, which was set at $3 \mathrm{~m}$ based on the results of the field investigation and analysis of the LiDAR data(Zhao et al., 2013; Dougherty and Lotufo, 2013).

\subsubsection{Individual tree crown algorithm:}

Marker-controlled watershed segmentation is a topological algorithm used in mathematical morphology. It has been widely and successfully applied in the field of remote sensing image processing, including the isolation of tree-level canopies based on high-resolution optical or LiDAR data(Wang et al., 2004; Chen et al., 2006; Zhao and Popescu, 2007). The markercontrolled watershed segmentation algorithm was employed to delineate the tree-level canopy from the CHM image in this study.

For marker-controlled watershed segmentation, appropriate CHM smoothing and potential treetop detection algorithms are key steps in obtaining optimum segmentation results. Based on the spatial distribution and shape features of the woody trees in the study area, a weighted mean filtering method was developed to smooth the CHM that had had the invalid values filled. Details of the algorithm are given below.

$$
\hat{C}_{\mathrm{w} / 2, \mathrm{w} / 2}=\sum_{i=1}^{w} \sum_{j=1}^{w} W_{i, j} * C_{i, j}
$$

where,

$$
\left\{\begin{array}{c}
W_{i j}=\frac{1 / T_{i j}}{\sum_{i=1}^{w} \sum_{j=1}^{w}\left(1 / T_{i j}\right) T_{i j}} \\
T_{i j}=S_{i j} * D_{i j} \\
S_{i j=\left|C_{i, j}-C_{\mathrm{w} / 2, \mathrm{w} / 2}\right| / a+b} \\
D_{i j}=\sqrt{(i-w / 2)^{2}+(j-w / 2)^{2}} / a+b
\end{array}\right.
$$

where, $\mathrm{w}$ is the width of the filtering window. $\mathrm{C}_{\mathrm{w} / 2, \mathrm{w} / 2}$ is the filtered height at the center of the window and $\mathrm{C}_{\mathrm{i}, \mathrm{j}}$ is the height of pixel $(i, j)$ in the filtering window; $W_{i, j}$ is the spatial weighting function-its value determines the degree to which neighboring pixels of similar height within a moving window (w) contribute height information for a predicted central pixel. $\mathrm{W}_{\mathrm{i}, \mathrm{j}}$ is defined by a normalized reverse distance, where the inverse of the combined height gradient and the spatial distance value, $T_{i, j}$, for a given pixel location is divided by the area-based inverse of $\mathrm{T}_{\mathrm{i}, \mathrm{j}}$ from the moving window. $\mathrm{S}_{\mathrm{i}, \mathrm{j}}$ is related to the gradient difference between $\mathrm{C}_{\mathrm{i}, \mathrm{j}}$ and the central pixel, and $\mathrm{D}_{\mathrm{i}, \mathrm{j}}$ is related to the distance between $C_{i, j}$ and the central pixel. $a$ and $b$ are two adjustment factors. Based on a large number of repeated trials, for optimum smoothing results, a, b and w were set as 1 , 1 , and 3 , respectively, in this study.

The potential treetops were then detected using the method of local maximum detection in the adaptive filtering window. The rules for detecting potential treetops were defined as follows.

$$
B_{\text {top }}=\left\{\begin{array}{c}
C_{s}(i, j) \in C_{\text {tree }} \\
C_{s}(i, j) \geq \operatorname{Max}\left(C_{s(3 \times 3)}(i, j)\right), C_{s}(i, j)>15 m \\
C_{s}(i, j) \geq \operatorname{Max}\left(C_{s(5 \times 5)}(i, j)\right), C_{s}(i, j)<15 m
\end{array}\right.
$$

where, $C_{s}(i, j)$ is the pixel $(i, j)$ in the CHM that has been smoothed by the weighted mean filtering method within the search window. $\mathrm{C}_{\mathrm{s}}(3 \times 3)$ represents a $3 \times 3$ window configuration and $C_{s}(5 \times 5)$ represents a $5 \times 5$ window configuration.

The size of the search window was determined based on the height of the trees. In this study area, it could clearly be seen that the high trees were concentrated within certain small areas, whereas the low trees were more dispersed. Therefore, if $\mathrm{C}_{\mathrm{s}}(\mathrm{i}, \mathrm{j})$ was higher than $15 \mathrm{~m}$, a small window $(3 \times 3)$ was used to search for the potential treetops; if $\mathrm{C}_{\mathrm{s}}(\mathrm{i}, \mathrm{j})$ was lower than $15 \mathrm{~m}$, a larger window $(5 \times 5)$ was employed to search for potential treetops. A pixel was determined as being a potential treetop if the height of the pixel was greater than or equal to the local maximum value within a specific window.

The traditional watershed segmentation was used to isolate different concave basins within the image. However, the crowns in the CHM were like salient hills and so the CHM was inverted before the watershed segmentation algorithm was applied, as illustrated by Eq. (5):

$$
C_{\text {close_R }}=\operatorname{Max}\left(C_{\text {close }}\right)-C_{\text {close }}
$$

where $\mathrm{C}_{\text {close }}$ is the value of $\mathrm{C}_{\text {filled }}$ smoothed by the morphological closing operator46 in order to avoid over-segmentation. $\mathrm{C}_{\text {close_}} \mathrm{R}$ is the inverted value of $\mathrm{C}_{\text {close }}$.

Based on the results of the potential treetop detection, the pixels in $\mathrm{C}_{\text {close_R }}$ located at the potential treetops were all marked as having a value of 0 . The thus marked $C_{\text {close_R }}$ was then processed using the watershed segmentation algorithm, as illustrated by Eq. (6). $C_{\mathrm{w}}$ is the final tree-level canopy area.

$$
C_{W}=\operatorname{Mask}\left(\text { Watershed }\left(C_{\text {close }}{ }_{R}, B_{\text {top }}\right), C_{\text {tree }}\right)
$$

3.1.3 Crown-level structural parameters calculation and validation:

The results of watershed segmentation usually differ from the actual shape of the tree crowns because of the rough surface of the CHM. The morphological opening operator was, therefore, applied to optimize the segmented image. Each of the optimized basins represented the area of a single tree crown. Within a defined basin, the local maximum pixel in the CHM that had had the invalid values filled was regarded as being the actual treetop and the height of the treetop was taken to represent the actual tree height. The average values of the widths in the north-south and east-west directions for each basin were taken as the crown diameter. Due to the lack of in-situ information regarding the positions of the individual trees, validation of tree structural parameters derived by LiDAR data was conducted at the plot scale. Within each plot, the average height and crown diameter of all the trees as derived from the LiDAR data was compared with data from in-situ measurements.

\subsection{Classification of tree species based on spectral and} shape features

There were a lot of shadows in the canopy and these had a big influence on the accurate extraction of reflectance spectra from the high-resolution images. To deal with this problem, the CASI aerial hyperspectral images were processed using a spectral normalization method(Leckie et al., 2003).

As there was no significant difference between the reflectance features of poplar and Chinese scholar tree, the shape characteristics related to their crown diameters and heights were analyzed. In our study area, the poplars are usually tall with small crowns, while the Chinese scholar trees have relatively large crowns but are shorter. Therefore, the ratio of height to crown diameter $\left(\mathrm{T}_{\mathrm{h} / \mathrm{c}}\right)$ is capable of distinguishing the poplars from the Chinese scholar trees. The threshold for $\mathrm{T}_{\mathrm{h} / \mathrm{c}}$ was set as 1.8 based on a number of trails.

\subsection{Accuracy assessment and comparative analysis}

The classification scheme, consisted of ash, maple, oak, and other species, was chosen based on our field survey with the study area. The total 867 trees were divided into a training data set (around 25\% for poplar but 50\% for other species) and a testing data set following the random sampling strategy (see 
Table 1). Classification accuracy was assessed based on the computation of the overall accuracy (OA), producer's accuracy (PA), user's accuracy (UA), and the Kappa (Kc) statistic (Petropoulos et al., 2012).

In order to conduct comparative analyses, both spectral-metrics and LiDAR-metrics classifications were carried out. With the spectral-metrics classification, the crown-scale spectrum and spectral indexes of an individual tree was extracted from the pixel at treetop location in the crown region, and then a c5.0 classifier was applied to classify the individual tree to a particular species. For LiDAR-metrics classification, crown shape index and Laser points distribution were implemented to generate a decision tree rules using C5.0.

Table 1. Ground reference data in number of trees used in the

\begin{tabular}{|c|c|c|c|}
\multicolumn{5}{c}{ classification. } \\
Tree species & $\begin{array}{c}\text { Total } \\
\text { number }\end{array}$ & $\begin{array}{c}\text { Training } \\
\text { samples }\end{array}$ & $\begin{array}{c}\text { Testing } \\
\text { samples }\end{array}$ \\
\hline Poplar tree & 556 & 139 & 417 \\
\hline Willow tree & 165 & 83 & 82 \\
\hline $\begin{array}{c}\text { Chinese } \\
\text { scholar tree }\end{array}$ & 146 & 73 & 73 \\
\hline
\end{tabular}

\section{RESULT AND DISCUSSION}

\subsection{Woody CHM}

Figure 3 shows the CHMs that were extracted using different methods. It can be seen that both the woody trees and buildings are present in the CHM extracted by the MCC method only, whereas only the woody trees are present in the CHM extracted by integrating the MCC method and the spectral index threshold as described in section 3.1.1. It was concluded that the method using both the MCC method and spectral index threshold was very suitable for areas of agroforestry.

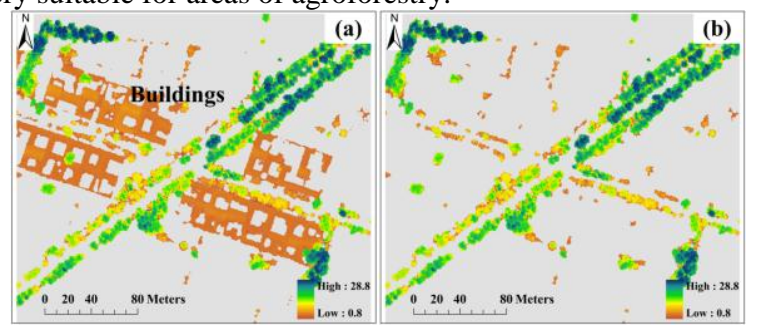

Figure 3. CHM extracted by only the morphological crown control method (a) and CHM extracted by integrating the morphological crown control method and CASI image (b).

\subsection{Individual tree segmentation}

It is well known that an appropriate CHM smoothing and treetop detection algorithms are key steps in obtaining optimum crown segmentation results. A CHM smoothing algorithm was proposed in this study to improve the accuracy of treetop detection. Figure 4 shows a comparison between the original CHM, the CHM smoothed by a median filter (Wang et al., 2004 )and the CHM smoothed by a weighted mean filter. It is obvious that the CHM smoothed by the weighted mean filter is smoother than that smoothed by the median filter and, at the same time, the height gradients between CHM cells are well preserved.

To evaluate the effect of the CHM smoothing algorithm, the same treetop detection algorithm and tree recognition algorithm were applied to different smoothed CHMs. It was found that the RMSE for the number of trees extracted using the CHM smoothed by a weighted mean filter was 4.86 trees, which is acceptable for estimating plot-level woody biomass density and lower than that for the median-filtered CHM (RMSE $=9.2$ trees), as illustrated in Figure 5. These results show that a weighted-mean filtered CHM can accurately measure the canopy surface morphology and, to some extent, improve the accuracy of individual tree segmentation for the sparse tree cover found in this study area.

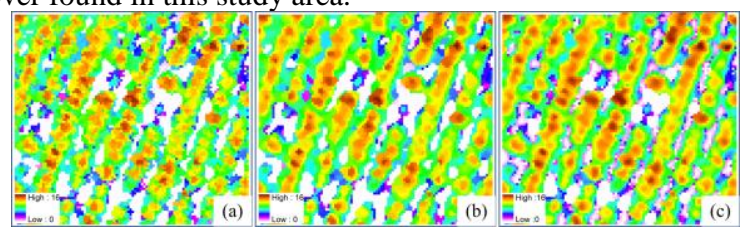

Figure 4. (a) Original CHM, (b) Median filter, (c) Weighted mean filter
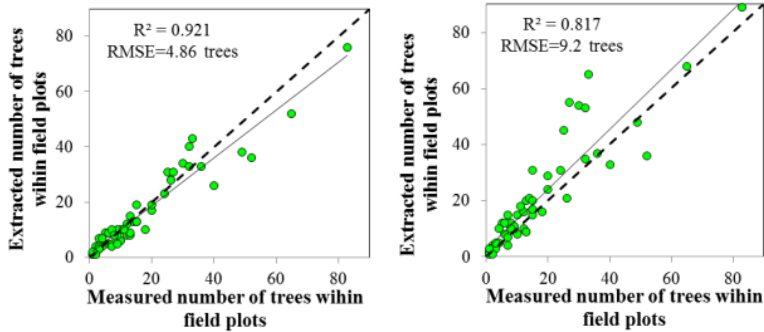

Figure 5. Extracted number of trees obtained using (a) the CHM smoothed by a weighted mean filter and (b) the CHM smoothed by a median filter

\subsection{Crown-level spectra calculation}

In this study, to extract the spectral features, $3 \times 3$ neighborhood regions centered on the treetops that had been detected by applying the treetop detection algorithm to the CHM image were selected from the spectrally normalized CASI imagery for each of the three tree species; the resulting average spectrum for each species is shown in Figure 6.

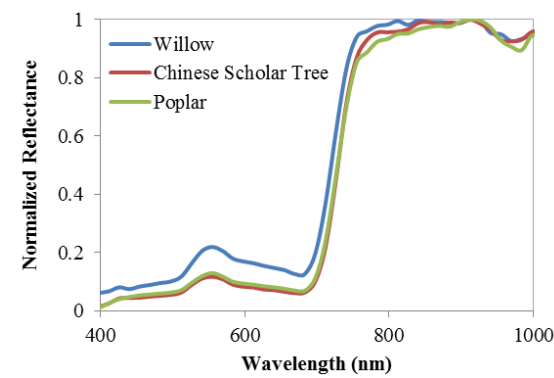

Figure 6. Normalized reflectance spectra of the three tree species.

\subsection{Crown-level structural parameters and validation}

Figure 7 shows the tree-level structural parameters (heights and crown diameters) extracted from CASI hyperspectral images and LiDAR data using a series of image processing algorithms. The black crosses show the positions of the treetops. The accuracy evaluation results are shown as scatter plots in Figure 8. The general accuracy of the extracted tree heights is acceptable- the RMSE is $1.79 \mathrm{~m}$. The accuracy of the extracted tree heights decreases as the tree height increases. The accuracy of the extracted crown diameters is not as high as that for the tree heights - the RMSE is $1.03 \mathrm{~m}$. The crown diameters are underestimated in most cases. The main reason for this is sheltering by adjacent crowns. 


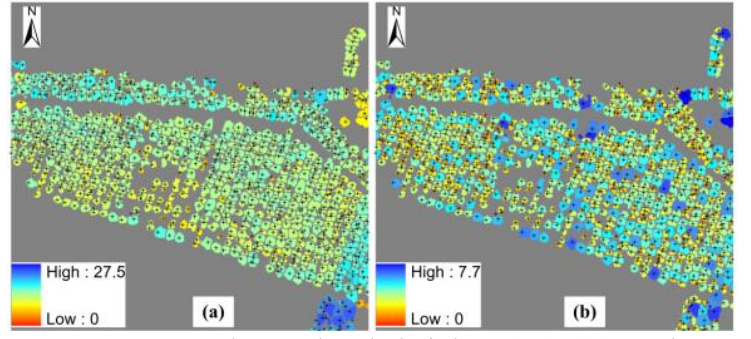

Figure 7. Extracted tree-level heights (m) (a) and crown diameters $(\mathrm{m})(\mathrm{b})$.
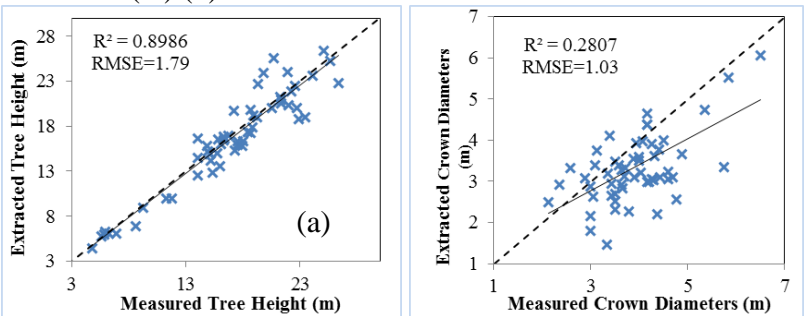

Figure 8. Comparison between average measured values of plotlevel tree structural parameters and estimates derived from LiDAR data: (a) tree heights, (b) crown diameters.

\subsection{Crown-level Tree species classification}

The crown-level tree species classification at crown-level was made according to the spectral and shape features described in section 3.2. The classification results for the four sub-regions are shown in Figure 9.

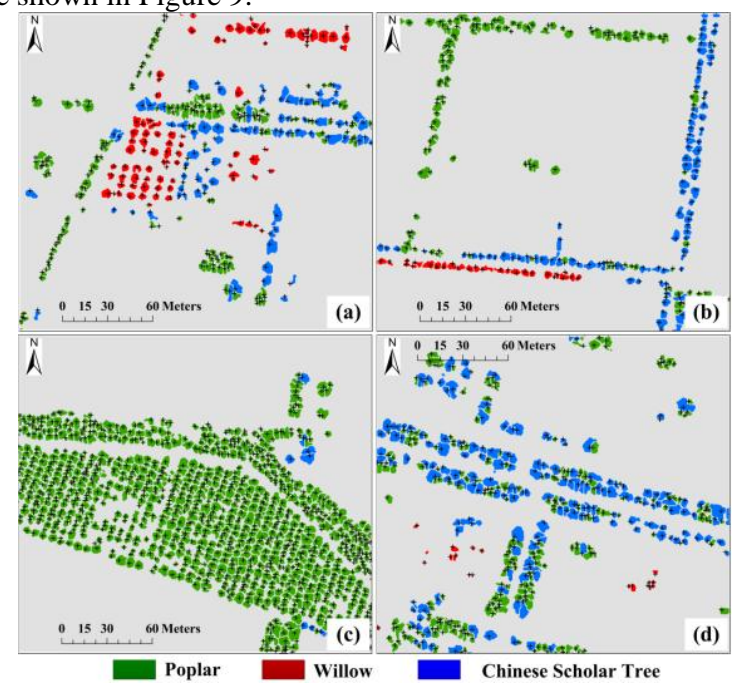

Figure 9. Tree species classification results for the four subregions in the study area

\subsection{Accuracy assessment}

In this paper, information about tree species and numbers of trees (572) in the testing data set (Table 1) acquired from in-situ investigations were used to validate the classification results based on a confusion matrix method.

In addition, tree methods including spectral-metrics, LiDARmetrics and LiDAR+spectral classification were carried out in order to conduct comparative analysis. The classification accuracies $(\mathrm{OA}$ and $\mathrm{Kc}$ ) of three classification methods are shown in the Figure 10.
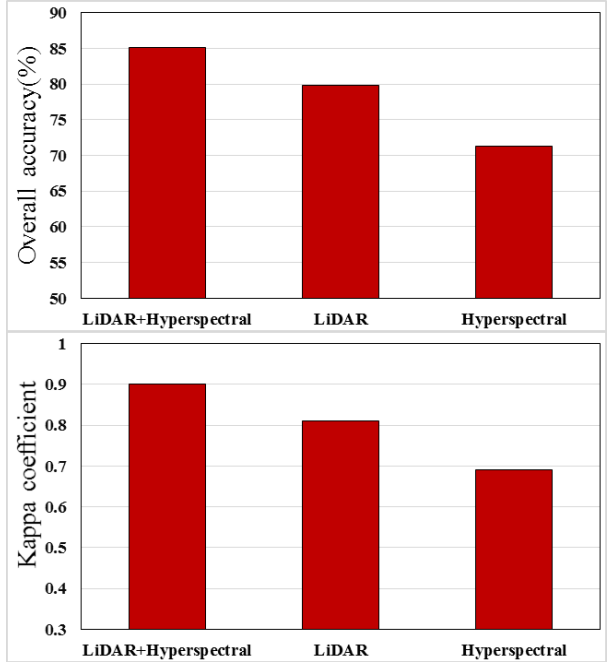

Figure 10. Accuracy assessment results of three different methods

The assessment result suggests that the developed classification strategy in this paper $(\mathrm{OA}=85.12 \%, \mathrm{Kc}=0.90)$ performed better than LiDAR-metrics method $(\mathrm{OA}=79.86 \%, \mathrm{Kc}=0.81)$ and spectral-metircs method $(\mathrm{OA}=71.26, \mathrm{Kc}=0.69)$ in terms of classification accuracy, which indicated that the advanced method of data processing and sensitive feature selection are critical for improving the accuracy of crown-level tree species classification. Unlike hyperspectral data, the LiDAR data acquired from semi-leaf-on conditions contain more interactions with the upper canopy making the characterization of the spatial arrangement of stems and branches better than under leaf-on situations (Yao et al., 2012). As a consequence, overall the LiDAR variables provided greater accuracy than the hyperspectral variables in this study.

\section{CONCLUSION}

In this study, we examined the capacity of airborne LiDAR and hyperspectral remote sensing data to accurately classify tree species in an agroforestry in the northwestern China using structural and spectral variables in isolation and in combination. The analysis revealed that a combination of both LiDAR and hyperspectral variables yielded an improved discrimination of tree species, with an overall accuracy of $85.12 \%$ compared to LiDAR $(79.86 \%)$ or hyperspectral $(71.26 \%)$ variables alone.

Although the results of crown-level tree species are satisfactory, further improvements may include exploration of spectral information. For example, applications of the spectral mixture analysis for pure crown-scale spectra calculation. Moreover, other experimental areas could be tested using this method in the further study. It could be concluded that in this paper individual crown structure features derived from LiDAR data, together with the developed crown-level spectra, may have potential for further improving tree species discriminations.

\section{ACKNOWLEDGEMENTS}

This work was supported by the Science foundation of central public-interest research institute (HKY-JBYW-2017-08), National Natural Science Foundation of China (51509102, 41701509), and Foundation of development on science and technology of Yellow River Institute of Hydraulic Research (HKF201709) 


\section{REFERENCES}

Li, C., Guan, W., Fan, X., Zhao, T., Chen, J., 2003. Assessment of landscape ecology of agricultural protection forest system at Beizang Town, Daxing County, Beijing. Journal of Forest Research, 14(2), pp. 135-140.

Zomer, R.J., Trabucco, A., Coe, R., Place, F., 2009. Trees on Farms: Analysis of the Global Extent and Geographical Patterns of Agroforestry. ICRAF Working Paper, No. 89, World Agroforestry Centre: Nairobi, Kenya.

Hernandez, G., Trabue, S., Sauer, T., Pfeiffer, R., Tyndall, J., 2012. Odor mitigation with tree buffers: Swine production case study. Agriculture Ecosystems \& Environment, 149, pp. 154163.

George, R., Padalia, H., Kushwaha, S., 2014. Forest tree species discrimination in western Himalaya using EO-1 Hyperion. International Journal of Applied Earth Observation and Geoinformation, 28, pp. 140-149.

Clark, M.L., Roberts, D.A., 2012. Species-level differences in hyperspectral metrics among tropical rainforest trees as determined by a tree-based classifier. Remote Sensing, 4, pp. $1820-1855$.

Lu, D., Weng, Q., 2004. Spectral mixture analysis of the urban landscape in Indianapolis with Landsat ETM+ imagery. Photogrammetric Engineering \& Remote Sensing, 70, pp. 1053-1062.

Yu, Q., Gong, P., Clinton, N., Biging, G., Kelly, M., Schirokauer, D., 2006. Object-based detailed vegetation classification with airborne high spatial resolution remote sensing imagery. Photogrammetric Engineering \& Remote Sensing, 72, pp. 799-811.

Dalponte, M., Bruzzone, L., Gianelle, D., 2012. Tree species classification in the Southern Alps based on the fusion of very high geometrical resolution multispectral/hyperspectral images and LiDAR data. Remote Sensing of Environment, 123, pp. 258-270.

Ke, Y., Quackenbush, L.J., Im, J., 2014. Synergistic use of QuickBird multispectral imagery and LIDAR data for objectbased forest species classification. Remote Sensing of Environment, 114, pp. 1141-1154.

Clark, M.L., Roberts, D.A., Clark, D.B., 2005. Hyperspectral discrimination of tropical rain forest tree species at leaf to crown scales. Remote Sensing of Environment, 96, pp. 375-398. Alonzo, M., Bookhagen, B., Roberts, D.A., 2014. Urban tree species mapping using hyperspectral and lidar data fusion. Remote Sensing of Environment, 148, pp. 70-83.

Shang, X., Chisholm, L.A., 2014. Classification of Australian native forest species using hyperspectral remote sensing and machine-learning classification algorithms. IEEE Journal of Selected Topics in Applied Earth Observations and Remote Sensing,7, pp. 2481-2489.

Zhang, C., Qiu, F., 2012. Mapping individual tree species in an urban forest using airborne lidar data and hyperspectral imagery. Photogrammetric Engineering \& Remote Sensing, 78, pp. 1079-1087.

Li, X., Cheng, G.D., Liu, S.M., Xiao, Q., Ma, M.G. et al., 2013. Heihe Watershed Allied Telemetry Experimental Research (HiWATER), Scientific Objectives and Experimental Design. Bulletin of the American Meteorological Society, 94(8), pp. 1145-1160.

Cheng, G., Li, X., Zhao, W., Xu, Z., Feng, Q., Xiao, S., Xiao, H., 2014. Integrated study of the water-ecosystem-economy in the Heihe River Basin. National Science Review, 1(3), pp. 413428.

Zhao, D., Pang, Y., Li, Z., et al.,2013. Filling invalid values in a lidar-derived canopy height model with morphological crown control. International journal of remote sensing, 34(13), pp. 4636-4654.

Dougherty,E.R., Lotufo,R.A., 2003. Hands-on morphological image processing. The International Society for Optical Engineering SPIE.

Wang, L., Gong, P., Biging, G.S.,2004. Individual tree-crown delineation and treetop detection in high-spatial-resolution aerial imagery. Photogrammetric Engineering \& Remote Sensing, 70(3), pp. 351-357.

Chen, Q., Baldocchi, D., Gong, P, et al.,2006. Isolating individual trees in a savanna woodland using small footprint lidar data. Photogrammetric Engineering \& Remote Sensing, 72(8), pp. 923-932.

Zhao, K., Popescu, S., 2007. Hierarchical watershed segmentation of canopy height model for multi-scale forest inventory. Proceedings of the ISPRS working group-Laser Scanning, pp. 436-442.

Leckie, D., Gougeon, F., Hill, D., et al.,2003. Combined highdensity lidar and multispectral imagery for individual tree crown analysis. Canadian Journal of Remote Sensing, 29(5), pp. 633-649.

Petropoulos, G.P., Arvanitis, K., Sigrimis, N., 2012. Hyperion hyperspectral imagery analysis combined with machine learning classifiers for land use/cover mapping. Expert Systems With Applications, 39, pp. 3800-3809.

Yao, W., Krzystek, P., Heurich, M., 2012. Tree species classification and estimation of stem volume and DBH based on single tree extraction by exploiting airborne full-waveform LiDAR data. Remote Sensing of Environment ,123, pp.368-380.

Revised March 2018 